

\title{
METODOLOGÍA PARA ELABORAR UNA CARTOGRAFÍA REGIONAL Y APLICAR ESTRATEGIAS BIOCLIMÁTICAS SEGÚN LA CARTA DE GIVONI
}

\author{
METHODOLOGY FOR A REGIONAL CARTOGRAPHY FOR THE \\ APPLICATION OF THE BIOCLIMATIC STRATEGIES OF THE GIVONI \\ CHARTER
}

\author{
FERNANDO DA CASA MARTÍN \\ Doctor en Arquitectura \\ Docente Catedrático del Departamento de Arquitectura \\ Universidad de Alcalá, Madrid, España \\ https://orcid.org/0000-0002-2728-4424 \\ fernando.casa@uah.es
}

\author{
FLAVIO CELIS D'AMICO \\ Doctor en Arquitectura \\ Docente Titular del Departamento de Arquitectura \\ Universidad de Alcalá, Madrid, España \\ http://orcid.org/0000-0003-3931-6056 \\ flavio.celis@uah.es
}

ERNESTO ECHEVERRÍA VALIENTE

Doctor en Arquitectura

Docente Titular del Departamento de Arquitectura

Universidad de Alcalá, Madrid, España

http://orcid.org/0000-0002-6826-5756

ernesto.echeverria@uah.es

\section{RESUMEN}

Se plantea como objetivo de la investigación expuesta la viabilidad de una metodología para cartografiar un territorio concreto, implementando las estrategias bioclimáticas necesarias para alcanzar el confort, según el diagrama de Givoni; herramienta muy útil para el diseño de edificios. Tal metodología se desarrolla en cuatro fases: I, obtención de la información climática; II, análisis de los datos climatológicos; III, selección de estaciones y datos mensuales, aplicación de la carta de Givoni e inicio del proceso de cartografiado; IV, establecimiento de la zonificación, y elaboración de mapas, con carácter mensual. Como resultado del trabajo, se obtiene un conjunto de mapas que indican las estrategias bioclimáticas adecuadas a cada territorio, en periodicidad mensual, para alcanzar el confort en los edificios. La metodología fue validada en un territorio concreto en España, utilizado como caso de estudio. En definitiva, la aportación original de la investigación es precisamente el desarrollo de la mencionada metodología, que permite elaborar una cartografía para un territorio determinado -mapa que convierte en una potente herramienta para el diseño bioclimático- y que, además, es susceptible de ser aplicada a cualquier territorio.

\section{Palabras clave}

arquitectura bioclimática, cartografía, climatología, territorio

\section{ABSTRACT}

The aim of the research is the feasibility of a methodology to map a specific territory, implementing the bioclimatic strategies necessary to achieve comfort, according to the Givoni diagram, as a very useful tool for building design. The methodology used is developed in four phases: I, obtaining the climatic information; II, analysis of climatological data; III, selection of stations and monthly data, application of Givoni letter and start of the mapping process; IV, establishment of zoning, and mapping, on a monthly basis. As a result of the application of the methodology, a set of maps is obtained that indicate the appropriate bioclimatic strategies for each territory, on a monthly basis, in order to achieve comfort in the buildings. The methodology has been validated in a specific territory

in Spain, used as a case study. The original contribution of the research is said methodology that allows to elaborate the cartography for a territory, that becomes a powerful tool for the bioclimatic design, and that is capable of being applied to any territory. 


\section{INTRODUCCIÓN}

\section{EL DISEÑO BIOCLIMÁTICO SEGÚN LAS ESTRATEGIAS DE GIVONI}

El diseño bioclimático es una herramienta para alcanzar niveles de confort térmico que tiene su origen en el estudio de la relación del hombre con el clima. Ya a principios del siglo XX, Houghten y Yagloglou trabajaron con el concepto de confort climático en su estudio "Determining lines of equal confort" (1923), formando parte de lo que hoy se conoce como ASHRAE (American Society of Heating, Refrigerating and Air-Conditioning Engineers). Este trabajo propuso ubicar zonas de confort en un diagrama psicométrico, relacionando humedad y temperatura, con base en el índice de temperatura efectiva. Muchas investigaciones se generan a partir de ese momento. Como evolución a dichos planteamientos se establecieron los llamados climogramas, diagramas bioclimáticos 0 cartas bioclimáticas, que son utilizados como herramientas para determinar las estrategias de diseño de espacios exteriores e interiores con el objetivo de alcanzar el confort térmico. Estas cartas permitieron vincular las condiciones climáticas del lugar con las estrategias pasivas $y / 0$ activas que se necesitan para lograr las condiciones de confort (Givoni, 1969).

En el desarrollo de estas herramientas se han realizado numerosas aportaciones orientadas a analizar las variables que intervienen en estos fenómenos y el modo en que lo hacen: Halawa y Van Hoof (2012), Mena, Rodríguez, Castilla y Arahal (2014), Dávila (2015), Larrumbide y Bedoya (2015), Kurbán y Cúnsulo (2017), y Esteves (2018). La mayor parte de ellas se abocan a las relaciones existentes entre las distintas variables térmicas y el confort humano, y su incidencia en la representación gráfica de dichas relaciones.

Los diagramas, o cartas bioclimáticas, más usados son el de Olgyay y el de Givoni, por ser estos aplicables de forma directa a los edificios y su entorno, de modo que se obtiene de ellos recomendaciones igualmente directas. El primero cuantifica las correcciones de los parámetros bioclimáticos para la obtención del confort humano en condiciones de exterior, sin ningún tipo de relación con el objeto arquitectónico. El segundo cuenta con las incidencias que puede producir la arquitectura en el clima y señala las cualidades que deben tener las edificaciones para conseguir la sensación de confort dentro de los mismos (Couret, Guzmán, Milián, García, y Salazar, 2015; Medina y Escobar, 2019).

La aplicación del diagrama de Givoni para el diseño bioclimático de los edificios está en su objetivo inicial. Ha sido utilizada tanto desde el ámbito del diseño de sistemas constructivos concretos (Balter, Ganem y Discoli, 2016), en casos concretos de edificios (Rodríguez, Nájera y Martín, 2018), como en la elaboración de manuales de arquitectura bioclimática para un territorio concreto (Pérez, Ladrón de

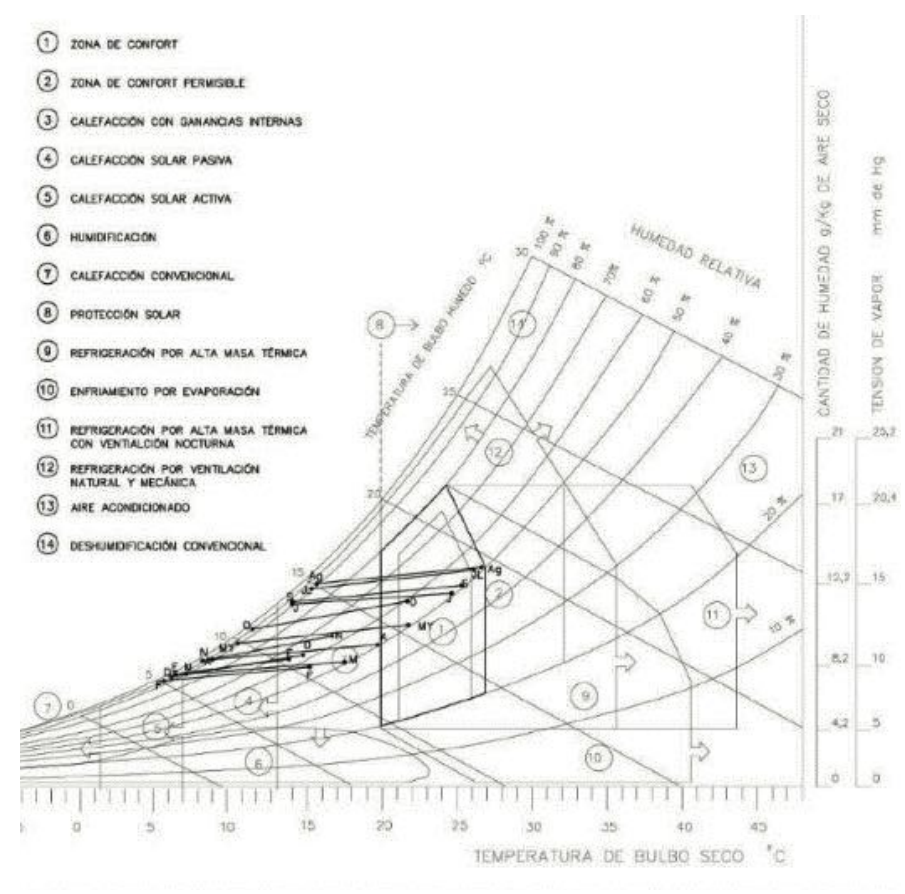

Figura 1. Diagrama de Givoni para las condiciones climáticas del periodo de recolección de datos: 2002-2016, en el observatorio meteorológico de Lourizán, Galicia, España. Fuente: Elaboración de autores.

Guevara y Boned, 2015). También hay referencias para Japón (Ooka, 2002), China (Gou, Li, Zhao, Nik y Scartezzini, 2015) e, incluso, Latinoamérica (Echeverría, García, Celis y Saelzer, 2019).

La carta bioclimática de Givoni se basa en el Índice de Tensión Térmica (ITS) para delimitar la zona de bienestar, y su aplicación es muy adecuada en climas cálidos de las regiones áridas. Este método tiene en cuenta las características de la construcción como modificadoras de las condiciones del clima exterior y recomienda el bienestar en el interior de las edificaciones.

Givoni (1969) propone una carta bioclimática en la que en el eje de abscisas se representa las temperaturas de bulbo seco, mientras en el eje de las ordenadas, la tensión parcial de vapor de agua contenido en el aire; las líneas curvas, psicométricas, representan la humedad relativa (Figura 1). Sobre la línea de máxima humedad (100\%) se ubica la temperatura de bulbo húmedo.

La representación del clima anual puede hacerse con las condiciones medias de cada mes. En el diagrama se delimitan varias zonas cuyas características de temperatura y humedad indican la conveniencia de utilizar unas determinadas estrategias de diseño en la edificación.

Cada una de estas zonas se puede observar en la Figura 1. Las estrategias de calefacción son sucesivas, mientras que, en las estrategias de refrigeración, se produce un solape y, por tanto, un abanico de posibilidades con las que se puede alcanzar la zona de confort. 


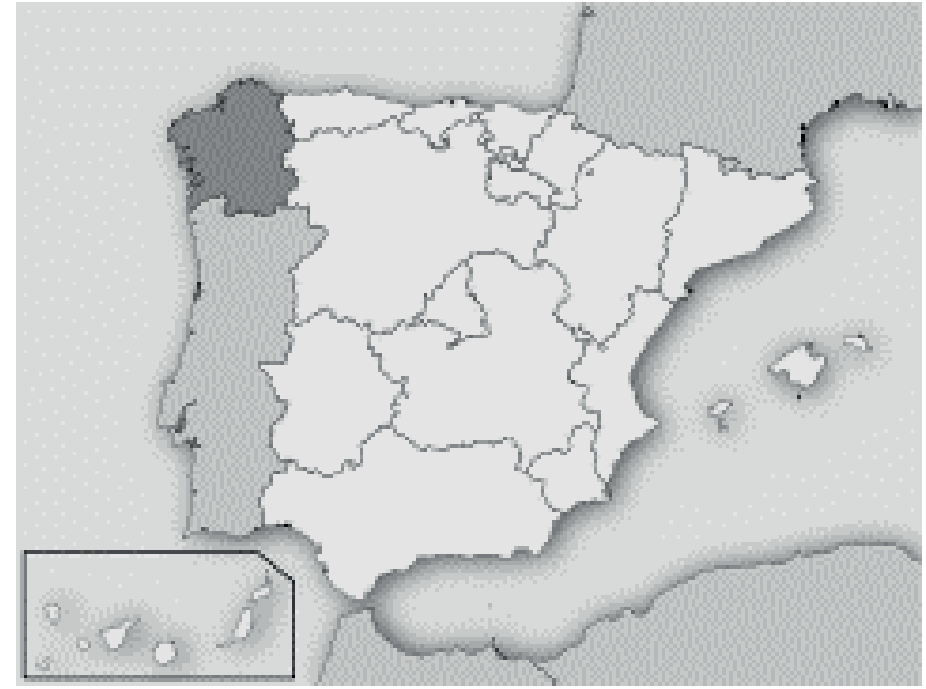

Figura 2. Localización geográfica de Galicia en la Península Ibérica (color más oscuro). Fuente: Instituto Geográfico Nacional (www.ign.es)

Las indicaciones obtenidas en el diagrama son orientativas, y no exclusivas. El diseñador tiene una información de los sistemas que son efectivos en ese lugar y en el tiempo analizado. Es a partir de esa información que el proyectista tomas las decisiones adecuadas. Habitualmente, lo recomendable es la adopción de medidas combinadas, que permitan el mejor aprovechamiento de los equipos instalados en las diferentes situaciones climáticas. Con ello se llega a verdaderas reducciones del consumo energético, además de la optimización del consumo de los equipos convencionales de climatización a instalar.

Hay que tener en cuenta también que la adopción de estrategias correctoras de las condiciones consideradas como insuficientes, favorece y abarata el uso de los sistemas convencionales. Así, si se necesita calefacción convencional, un buen comportamiento pasivo disminuirá la cantidad de energía que se gaste en ella (Da Casa, 2000).

El problema surge cuando para poder aplicar esta herramienta al proceso de diseño, la condición previa es disponer de los datos climatológicos correspondientes a la localización del edificio para integrarlos en el diagrama de Givoni y, de esa forma, conocer las estrategias bioclimáticas necesarias para corregir los parámetros de disconfort. La dificultad más habitual es, precisamente, obtener información concreta de la ubicación, que sea fiable. En muchas ocasiones no existe, lo que obliga a complejos procesos de investigación (Corral, García y Romero, 2018). En otros casos, los archivos de datos climáticos para la simulación son muy generalistas y simplificados (con el fin de facilitar su manejo), sin tener en cuenta factores específicos del lugar, como sobrecalentamientos locales o microclimas, ni tampoco los efectos del cambio climático (Luciani, Velasco y Hudson, 2018).

Se presenta, en suma, la dificultad de utilizar esta herramienta de forma global, en cualquier territorio, para alcanzar los beneficios de un diseño sostenible. Ante esto, y como punto de partida de la investigación, se considera la necesidad de responder a las siguientes cuestiones: ¿Es posible disponer de datos climatológicos generalizables para un uso inmediato?, ¿es posible generar un modelo de aplicación a partir de los diagramas de Givoni?, ¿es posible extraer de ellos una interpretación básica de carácter territorial? Y, lo más importante, ¿es posible cartografiar todo ello?

La obtención de respuestas a estas preguntas es la base del objetivo de esta investigación, que propone desarrollar una metodología óptima para elaborar una "cartografía regional bioclimática", de un territorio concreto, utilizable por cualquier técnico en el diseño de edificios, en la que se disponga de la información necesaria para adoptar los criterios y estrategias más adecuadas, basándose en la carta bioclimática de Givoni.

Entre los estudios relacionados con el tema, se puede citar los que abordan la representación cartográfica de bioclimas (Marco, Sariñena, López, M.S. y López, M.L., 2016) o de parámetros, como la radiación solar (Díaz, Montero y Mazorra-Aguiar, 2018). Hay también algunos intentos de grafiar la zonificación climática de referencia del Código Técnico de la Edificación de España. No se cuenta con ninguna referencia a la generación de cartografías específicas referidas a las estrategias bioclimáticas de diseño, más allá de una propuesta metodológica expuesta por Da Casa (2000).

El desarrollo y aplicación de estas estrategias facilita la consecución de los objetivos ODS, así como el de todas las normativas de eficiencia energética, tanto las de reducción de consumos, como las del aumento de la optimización de los edificios desde su propio diseño. Disponer de esta nueva herramienta (usando los recursos disponibles, sin tener que realizar una inversión, en caso de que se trate de una iniciativa pública), genera un gran interés, dada la rentabilidad de su planteamiento y su alto potencial de beneficios para un territorio concreto.

\section{METODOLOGÍA}

En el proceso de investigación se ha establecido una metodología clara, concreta y completa, para la elaboración de este tipo de cartografías. Con el propósito de comprobar su factibilidad se utilizó como sistema de validación un ejemplo aplicado a un territorio concreto, a modo de experiencia piloto. El territorio seleccionado fue la Comunidad Autónoma de Galicia, en España (Figura 2).

Se establece, en este contexto, cuatro fases principales:

- Fase I. Obtención de la información climática disponible actualizada del ámbito de estudio. 
- Fase II. Análisis de los datos climatológicos.

- Fase III. Implantación de estaciones y datos mensuales según estrategias bioclimáticas de Givoni. Inicio del proceso de cartografiado.

- Fase IV. Zonificación, y establecimiento de los "mapas" de estrategias bioclimáticas de Givoni, con carácter mensual.

A continuación, se profundiza en cada una de estas etapas.

\section{FASE I. OBTENCIÓN DE LA INFORMACIÓN CLIMÁTICA DISPONIBLE ACTUALIZADA DEL ÁMBITO DE ESTUDIO}

Para la obtención de datos climáticos específicos de la zona de estudio, se dispuso de una red de observatorios meteorológicos con los datos que se precisan (al menos, temperaturas medias, máximas y mínimas, y humedades relativas medias, máximas y mínimas). La fuente de información establecida fue la red de observatorios meteorológicos de la Xunta de Galicia (www.meteogalicia.es), que posee un total de 101 observatorios distribuidos de forma generalizada por toda su geografía (Figura 3).

Respecto del periodo mínimo de datos meteorológicos a recopilar, se planteó que este debía ser el suficiente como para ser referentes del clima real (habitualmente se consideran 30 años). Ahora bien, hay que tener en cuenta que la extensión y digitalización de los observatorios no permite alcanzar en la mayoría de los casos dicha cuantía.

A ello hay que sumar el potencial efecto de la situación de cambio climático actual, en la que puede aceptarse como modelo la existencia de un ligero aumento de la temperatura (Solanki, Schüssler y Fligge, 2000; Solanki, Usoskin, Kromer, Schüssler y Beer, 2004), sobre todo en invierno, y un desplazamiento de las precipitaciones invernales hacia la primavera, lo que conlleva un incremento de la oceanidad y de la templanza (no una tropicalización). Afecta a las instalaciones existentes en los edificios (Sánchez, Rubio, Marrero, Guevara y Canivell, 2017), así como a otros ámbitos (Enríquez, Díaz, Martín y Santos, 2017). Esto afecta de forma directa a la aplicación de las estrategias en el diseño pasivo (Rubio, 2015).

Por todo lo anterior, se estima como aceptable referencia un rango de tiempo en torno a los 15 años (periodo representativo del cambio). En los casos en los que se disponían de menores periodos, los datos se comprobaron en términos de su coherencia con el resto de los observatorios y se utilizaron de forma complementaria.

En el caso de Galicia, los observatorios de primera implantación datan del año 2000, lo que implica un período de más de 15 años. Además, todos los observatorios consultados cuentan con los datos precisos para la aplicación de la metodología considerada en este trabajo.

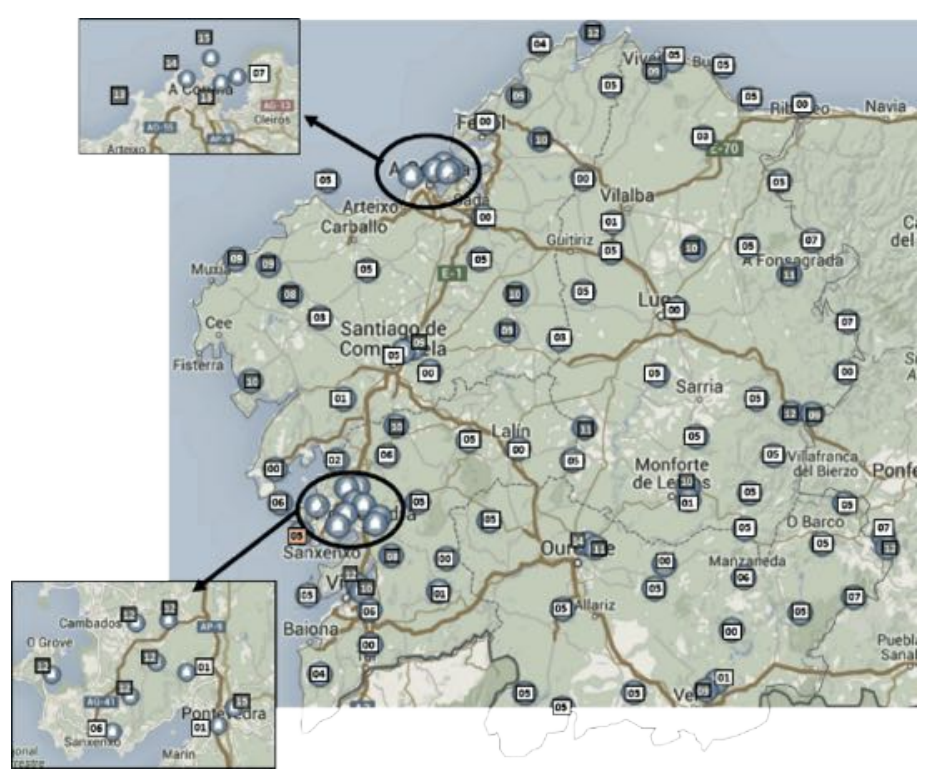

Figura 3. Estaciones meteorológicas disponibles de Galicia con fecha de implantación. Fuente: Elaboración de los autores en base a imágenes extraídas de www.meteogalicia.es.

Así, se seleccionaron 55 observatorios distribuidos homogéneamente en el territorio, dando prioridad a los de mayor antigüedad. Tal selección se efectuó en dos fases: 33 observatorios en la primera y otros 22 en la segunda (Tabla 1). Se recopilaron los datos de los parámetros climáticos necesarios para la elaboración de la carta bioclimática de Givoni de todo el período disponible de cada observatorio con carácter mensual, esto es, datos mensuales de temperaturas máximas, temperaturas mínimas, media de las temperaturas máximas, y media de las mínimas, así como datos de la humedad relativa media, máxima y mínima.

Para que la distribución geográfica de los observatorios a consultar fuera homogénea, el proceso de selección se realizó, como ya se indicó, en dos fases: en la primera se escogió a los de mayor prioridad (33 observatorios), y la segunda se complementó con otros 22 observatorios, a efecto de tener una cobertura lo más completa posible del territorio de análisis.

Con el fin de comprobar si era válida la exhaustividad del estudio, con 55 observatorios testados, es de referir que, en 2010, la "Guía técnica de condiciones climáticas exteriores de proyecto", elaborada por la Asociación Técnica Española de Climatización y Refrigeración (ATECYR) para el Instituto para la Diversificación y Ahorro de la Energía (IDAE), se basaba en los datos de sólo 6 Estaciones Meteorológicas para determinar el clima en Galicia. El objetivo de este estudio era promocionar la eficiencia en el uso final de la energía en los edificios. A partir de ese antecedente, se da por validado el número de puntos de estudio. 


\begin{tabular}{|c|l|l|l|c|}
\hline \multicolumn{3}{|c|}{ OBSERVATORIOS PRINCIPALES CONSULTADOS (1a fase) } \\
\hline $\begin{array}{c}\text { No } \\
\text { clave }\end{array}$ & Denominación & Provincia & Municipio & $\begin{array}{c}\text { Año } \\
\text { implantación }\end{array}$ \\
\hline 1 & Castro Vicaludo & Pontevedra & Oia & 2004 \\
\hline 2 & Monte Aloia & Pontevedra & Tui & 2000 \\
\hline 3 & Queimadelos & Pontevedra & Mondariz & 2001 \\
\hline 4 & Entrimo & Ourense & Entrimo & 2005 \\
\hline 5 & Gandarela & Ourense & Celanova & 2005 \\
\hline 6 & Baltar & Ourense & Baltar & 2005 \\
\hline 7 & O Invernadeiro & Ourense & Vilariño de Conso & 2000 \\
\hline 8 & Xares & Ourense & A Veiga & 2007 \\
\hline 9 & Lourizán & Pontevedra & Pontevedra & 2001 \\
\hline 10 & Amiudal & Ourense & Avión & 2005 \\
\hline 11 & Alto do Rodicio & Ourense & Maceda & 2000 \\
\hline 12 & San Xoán de Río & Ourense & San Xoán de Río & 2005 \\
\hline 13 & As Petarelas & Ourense & Rubias & 2005 \\
\hline 14 & Corrubedo & A Coruña & Ribeira & 2000 \\
\hline 15 & Muralla & A Coruña & Lousame & 2001 \\
\hline 16 & Sergude & A Coruña & Boqueixon & 2000 \\
\hline 17 & Serra do Faro & Pontevedra & Rodeiro & 2005 \\
\hline 18 & Bóveda & Lugo & Bóveda & 2005 \\
\hline 19 & Courel & Lugo & Folgoso & 2005 \\
\hline 20 & Fontecada & A Coruña & Santa Comba & 2003 \\
\hline 21 & Río do Sol & A Coruña & Coristanco & 2005 \\
\hline 22 & Melide & A Coruña & Melide & 2003 \\
\hline 23 & Campus Lugo & Lugo & Lugo & 2000 \\
\hline 24 & Ancares & Lugo & Cervantes & 2000 \\
\hline 25 & Malpica & A Coruña & Malpica & 2005 \\
\hline 26 & Mabegondo & A Coruña & Abegondo & 2000 \\
\hline 27 & Guitiriz & Lugo & Guitiriz & 2000 \\
\hline 28 & Pol & Lugo & Pol & 2005 \\
\hline 29 & CIS Ferrol & A Coruña & Ferrol & 2000 \\
\hline 30 & Punta Candieira & A Coruña & Cedeira & 2004 \\
\hline 31 & Serra da Faladoira & A Coruña & Ortigueira & 2005 \\
\hline 32 & Fragavella & Lugo & Abadín & 2003 \\
\hline 33 & Pedro Murias & Lugo & Ribadeo & \\
\hline & & & \\
\hline
\end{tabular}

\begin{tabular}{|c|l|l|l|c|}
\hline \multicolumn{5}{|c}{ OBSERVATORIOS CONSULTADOS en 2a fase } \\
\hline $\begin{array}{c}\text { No } \\
\text { clave }\end{array}$ & Denominación & Provincia & Municipio & $\begin{array}{c}\text { Año } \\
\text { implantación }\end{array}$ \\
\hline A & A Pontenova & Lugo & A Pontenova & 2005 \\
\hline B & O Xipro & Lugo & A Fonsagrada & 2007 \\
\hline C & Ventosa & Lugo & Navia de Suarna & 2007 \\
\hline D & Caldas de Reis & Pontevedra & Caldas de Reis & 2006 \\
\hline E & Pereira & Pontevedra & Forcarei & 2005 \\
\hline F & Mouriscade & Pontevedra & Lalín & 2000 \\
\hline G & Ourense & Ourense & Ourense & 2011 \\
\hline H & Marroxo & Lugo & Monforte & 2001 \\
\hline I & Serra do Eixe & Ourense & O Barco de Valdeorras & 2005 \\
\hline J & Cabeza de Manzaneda & Ourense & Manzaneda & 2006 \\
\hline K & Viana do Bolo & Ourense & Viana do bolo & 2005 \\
\hline L & Fornelos de Montes & Pontevedra & Fornelos de monte & 2000 \\
\hline M & Penedo do Galo & Lugo & Viveiro & 2005 \\
\hline N & Portomarín & Lugo & Portomarín & 2005 \\
\hline O & Abradelo & Lugo & Samos & 2005 \\
\hline P & Verín-Vilamaior & Ourense & Verin & 2001 \\
\hline Q & Corón & Pontevedra & Vilanova de Arousa & 2002 \\
\hline R & Rebordelo & Pontevedra & Cotobade & 2005 \\
\hline S & Ons & Pontevedra & Bueu & 2005 \\
\hline T & Illas Cíes & Pontevedra & Vigo & 2005 \\
\hline U & Camariñas & A Coruña & Camariñas & 2009 \\
\hline V & Lira & A Coruña & Carnota & \\
\hline & & & & \\
\hline & & & & \\
\hline & & & & \\
\hline & & & & \\
\hline & & & & \\
\hline & & & & \\
\hline & & & & \\
\hline & & & & \\
\hline & & & & \\
\hline & & & \\
\hline
\end{tabular}

Tabla 1. Relación de observatorios seleccionados en el estudio (se indica el año de implantación). Fuente: Elaboración de los autores

\section{FASE II. ANÁLISIS DE LOS DATOS CLIMATOLÓGICOS}

Una vez recopilada toda la información climatológica, se consideró como primer paso proceder a la homogeneización de los datos obtenidos de cada observatorio, según los modelos existentes (Arava, 2014; Cartaya, Zurita y Montalvo, 2016). Se adquirieron, de este modo, tablas individualizadas de cada uno de los observatorios con los datos de carácter mensual (en todo el periodo disponible) de temperaturas máximas y mínimas, medias de máximas y mínimas, y humedades relativas medias, máximas y mínimas. Esta información permitió el análisis de su evolución y el análisis comparativo con las temperaturas extremas. Se debe indicar que se filtraron los datos que respondían a situaciones de fallas del equipo o a deficiencias en la adquisición de estos.

Los datos medios por mes de cada parámetro se introdujeron en el climograma de Givoni, y con el resultado emanado se establecieron las estrategias bioclimáticas con carácter mensual, tanto las de refrigeración, como las de calefacción, en los meses requeridos.

Como es conocido, las estrategias de Givoni para calefacción son progresivas según desciende el dato de la temperatura. Ello hace posible emplear como herramienta hojas de cálculo para presentar los datos mensuales resultantes, pudiéndose entonces definir la estrategia límite de calefacción que permita alcanzar el confort, para cada mes y en cada zona.

Con respecto a las estrategias de refrigeración, la aplicación no resulta tan directa, ya que las estrategias se solapan, y su evolución no es tan clara como en lo referente a calefacción, al depender no solo de la variación de la temperatura, sino de la relación con la humedad relativa ambiental. En este tipo de estrategias se precisa la aplicación directa del diagrama de Givoni, para establecer resultados. Hay que contemplar que en la mayoría de las ocasiones se dispondrán de varias estrategias, que incluso pueden ser adoptadas de forma simultánea (Guzmán, Cano y Roset, 2019).

Con los datos obtenidos, se prepararon tablas y diagramas correspondientes a cada observatorio, en las que se indicó el tipo de estrategia requerida para alcanzar las condiciones de confort en cada mes, correspondientes a calefacción y a refrigeración de forma independiente. 

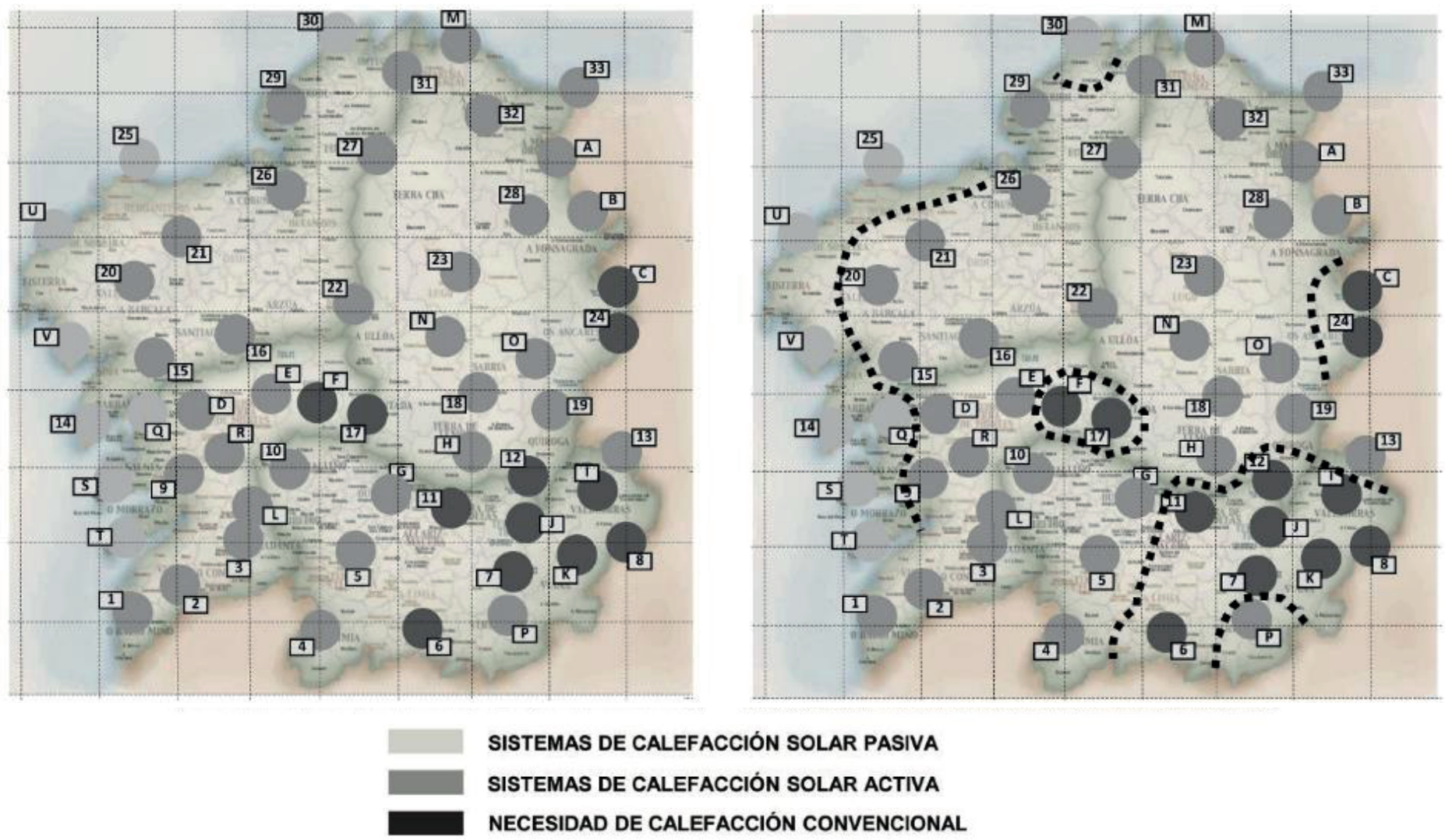

Figura 4. Ejemplo ilustrativo del proceso inicial de cartografiado correspondiente a las necesidades de calefacción del mes de febrero. Fuente. Elaboración de los autores.

\section{FASE III. IMPLANTACIÓN DE ESTACIONES Y DATOS MENSUALES SEGÚN ESTRATEGIAS BIOCLIMÁTICAS DE GIVONI. INICIO DEL PROCESO DE CARTOGRAFIADO}

El primer paso de esta fase fue el traspaso sobre un mapa del territorio, de los datos obtenidos de forma individualizada en cada observatorio. Se plasmó gráficamente con código de color según la diferente estrategia necesaria para cada mes en la localización geográfica de cada observatorio. De esta manera, se fue disponiendo de un mapa por mes, con la totalidad de los puntos seleccionados y codificados. Se realizaron de forma independiente mapas para las estrategias de calefacción y las de refrigeración.

El segundo paso consistió en llevar a cabo un primer análisis con el fin de comprobar la existencia de zonas de singularidad, por contraste entre resultados. Se efectuaron análisis complementarios de nuevos observatorios para complementar la información y poder definir mejor la complejidad de las zonas límites. Entonces, se pudo observar la existencia de zonas de similar comportamiento.

En el tercer paso de esta fase se procedió a la agrupación zonal de los observatorios, cuyos datos implican la utilización de estrategias de diseño similares. Se establecieron gráficamente unos límites iniciales de cada una de las zonas de carácter territorial que tenían un comportamiento similar.
Valga, a modo meramente ilustrativo de lo expuesto, la Figura 4, en la que se muestra, a la izquierda, un posicionamiento de datos $y$, a la derecha, la primera delimitación zonal.

\section{FASE IV. ZONIFICACIÓN Y ESTABLECIMIENTO DE LOS "MAPAS" DE ESTRATEGIAS BIOCLIMÁTICAS DE GIVONI, CON CARÁCTER MENSUAL}

Una vez establecido el primer esbozo de mapas mensuales con los resultados en forma de puntos de datos, se analizaron los resultados en la delimitación entre zonas de diferente caracterización. Fue posible observar las divergencias - discordancias, y se detectaron las zonas de dudosa delimitación, o zonas en las que se presentaba algún comportamiento inicialmente incoherente.

En ese momento, se determinó la necesidad de ampliar el número de observatorios a estudiar, incorporando los indicados en la Tabla 1, como $2^{\text {a }}$ fase. En los casos en los que no se disponía de datos fiables de algún observatorio en dicha zona, se procedió a la interpolación, con base en la distancia efectiva y teniendo en cuenta la diferencia de altitud y de latitud entre observatorios. En algunas zonas puntuales se introdujo el factor orográfico y el régimen de vientos locales.

Tras modificar y ajustar las delimitaciones iniciales, se obtuvo la zonificación cartografiada, con carácter mensual, 

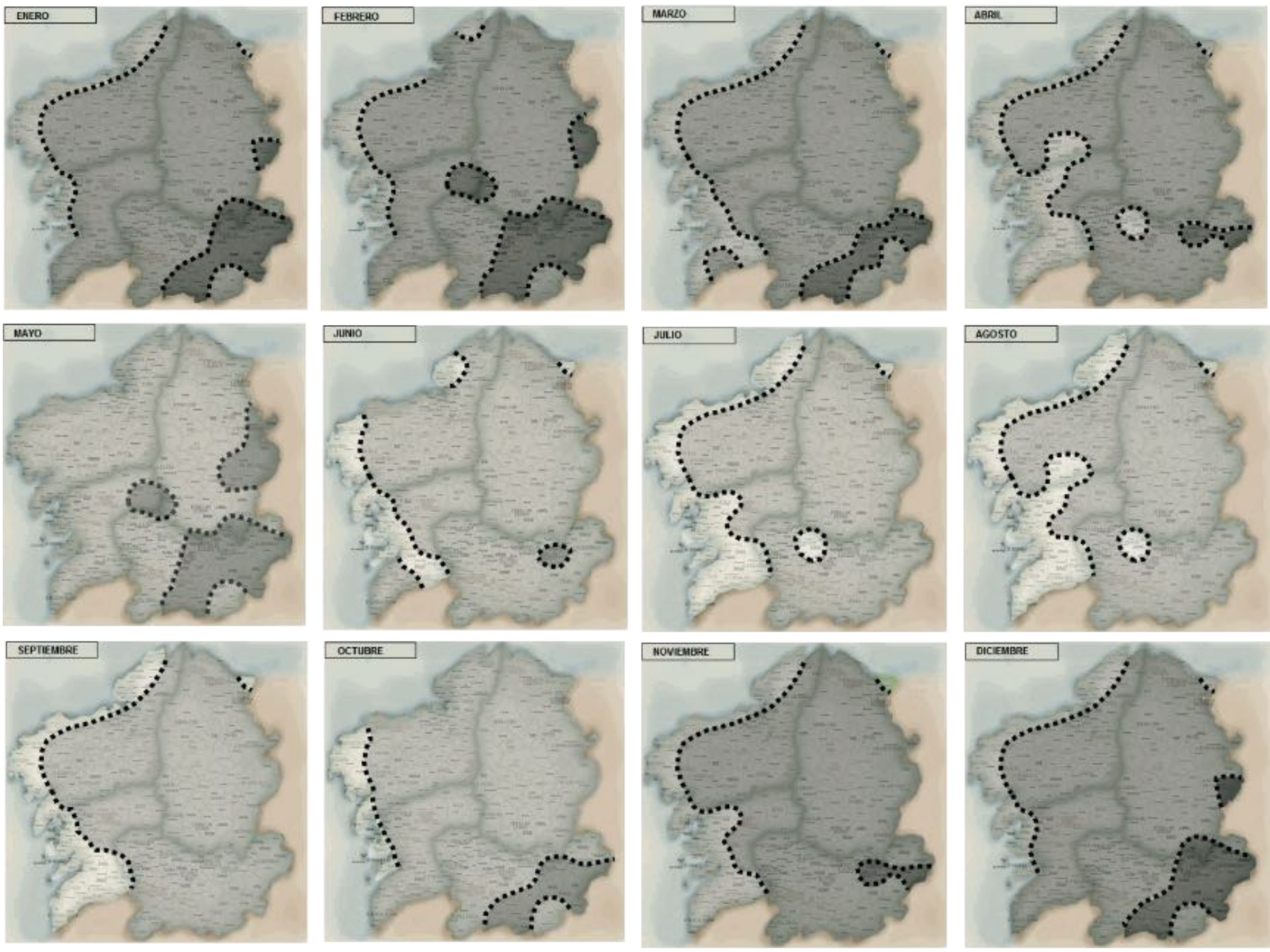

CONFORT POR GANANCIAS INTERNAS

SISTEMAS DE CALEFACCIÓN SOLAR PASIVA

SISTEMAS DE CALEFACCIÓN SOLAR ACTIVA

NECESIDAD DE CALEFACCIÓN CONVENCIONAL

Figura 5. Cartografía estrategias bioclimáticas para calefacción en Galicia para cada mes del año, según Givoni. Fuente: Elaboración de los autores.

de las estrategias de calefacción y de las requeridas para refrigeración (de forma independiente), según Givoni, con miras a lograr el confort térmico en el territorio estudiado.

Respecto a las estrategias de calefacción, el trabajo generó 12 mapas, uno por mes, ya que el análisis de los datos evidenció la necesidad de adoptar estrategias de calefacción durante todo el año. Así, en cada mes se señalaron las zonas diferenciables con distinta codificación.

La consideración de las situaciones extremas no se tuvo en cuenta en esta fase, sin perjuicio de que ello pueda incluirse en estudios posteriores. Ahora bien, tales situaciones no deben ser consideradas como base del diseño, ya que supondrían sobredimensionamientos excesivos que podrían generar situaciones irregulares.

En cuanto a las estrategias de refrigeración, se actuó de manera similar, plasmando mapas en cada uno de los meses en los que se presentaban este tipo de necesidades. Se planteó, al efecto de la investigación, establecer tantas agrupaciones de estrategias como se encontraban, según los solapes de la propia distribución del diagrama de Givoni.

Se decidió considerar las situaciones extremas para el caso de refrigeración, puesto que esos datos podrían ser útiles como complemento de la información de los parámetros medios, en la medida en que dan indicios de estados extremos de disconfort.

\section{RESULTADOS Y DISCUSIÓN}

Como resultado de la aplicación de la metodología propuesta, se comprobó la viabilidad de cartografiar las necesidades de estrategias de climatización de un territorio. En efecto, se obtuvieron 12 mapas, uno por mes, para las estrategias de calefacción (Figura 5). 

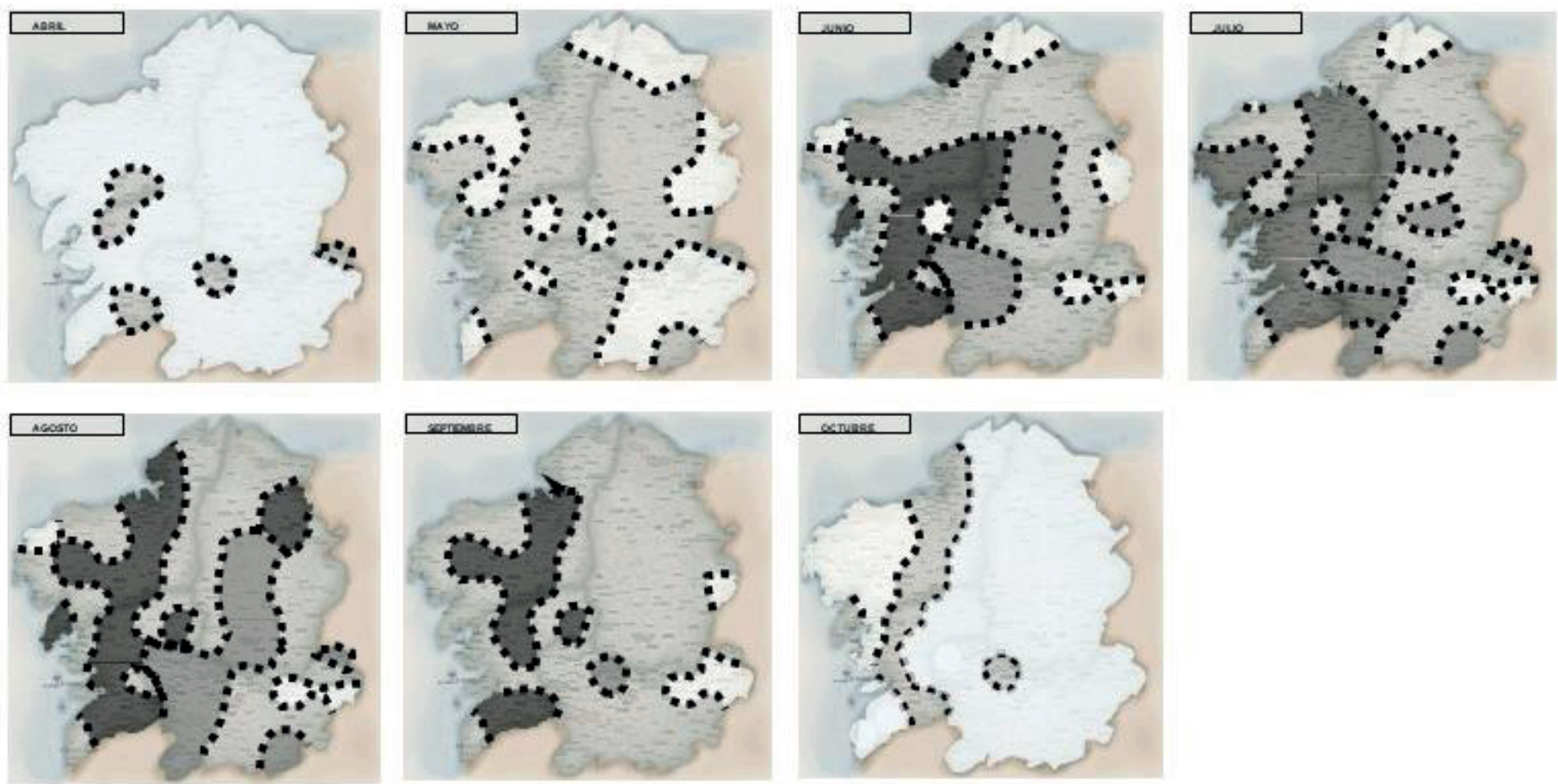

CONFORT. SIN NECESIDAD DE REFRIGERACIÓN

NECE SIDAD DE ALTA MASA INERCIA TÉRMICA Y VENTILACIÓN NOCTURNA

NECESIDAD DE ESTRATEGIAS DE VENTILACION

NECESIDAD DE AIRE ACONDICIONADO

Figura 6. Cartografía estrategias bioclimáticas para refrigeración en Galicia, para situación de temperaturas máximas, desde abril a octubre, según Givoni. Fuente: Elaboración de los autores.

En el caso de las estrategias de refrigeración, al aplicar los datos de temperaturas (máximas y mínimas) y humedades relativas medias (máximas y mínimas), se presentó una circunstancia particular, y es que lo habitual es que sean factibles varias estrategias, que pueden ser utilizadas de forma individual o simultánea. Pero el análisis de la evolución de dichas estrategias no fue aquí tan claro como en las correspondientes a los requerimientos de calefacción, fundamentalmente por el propio carácter no progresivo en las estrategias del diagrama de Givoni. Esto complicó la codificación, al producirse una mayor diversificación de puntos. Sin embargo, se fue comprobando en la práctica que al final la diversificación no es tan amplia para cada climograma.

Por otra parte, se observó que solo en 5 observatorios dispersos se indica alguna necesidad de refrigeración en algún mes, quedando prácticamente la totalidad del territorio en estado de confort (recordemos que con los datos medios mensuales).

Ante esta singularidad, se consideró interesante conocer el cartografiado de las necesidades de refrigeración en situación de temperaturas máximas, de modo que se pudo complementar la información del proceso de adopción de estrategias bioclimáticas en el diseño. Al aplicar el método en estas circunstancias, las estrategias de refrigeración se dataron desde abril hasta octubre, obteniéndose 7 mapas (Figura 6).
Frente a estos resultados es pertinente presentar la discusión llevada a cabo, que se enfocó en responder las preguntas relacionadas con tres aspectos principales: la fiabilidad del resultado obtenido, la aplicabilidad de la cartografía presentada, y la capacidad de extrapolar esta metodología a otros territorios.

\section{PUNTO 1 DE DISCUSIÓN: FIABILIDAD DEL RESULTADO OBTENIDO}

A la hora de responder a la pregunta "¿es fiable la información obtenida?", se vuelve esencial reflexionar sobre la propia definición de "fiabilidad". Si por tal término se entiende el grado de consistencia, estabilidad y ausencia de errores en los datos y su uso, se debe partir del origen de los datos utilizados. La fuente de los datos climáticos es de toda solvencia. Se trata de una red de observatorios meteorológicos de una administración pública, la Xunta de Galicia de acceso público de forma digital (www. meteogalicia.es). Los datos aplicados responden a la realidad de cada localización, los cuales fueron recogidos de forma continuada y digital.

La utilización de un periodo de recopilación de datos de 15 años permite establecer una consideración del clima real, atendiendo a la evolución producida y a la repercusión del cambio climático. Por tanto, los resultados basados en el uso de dichos datos pueden considerarse fiables. 
El grado de precisión de los mapas resultantes responde al carácter medio de los parámetros climatológicos utilizados, siendo ajustado para cualquiera de las aplicaciones para las que pueden utilizarse. El ajuste realizado en la delimitación entre zonas responde a dicho carácter con la interpolación entre observatorios, pero teniendo en cuenta la distancia efectiva entre ellos, la diferencia de altitud, e incluso la orografía intermedia de dicha zona. De este modo, se puede considerar haber obtenido una precisión equilibrada y fiable.

Ahora bien, si por fiabilidad se entiende la "probabilidad de un buen funcionamiento de algo" (definición de la Real Academia Española), se debe indicar que se ha realizado la comprobación de la coherencia de los mapas obtenidos, con la delimitación climática de la Normativa Española del Código Técnico de la Edificación (Da Casa, Echeverría y Celis, 2017), con un resultado positivo. No obstante, es patente que cada una de estas zonificaciones responde a objetivos diferentes y que la zonificación resultante de la metodología aplicada, al no limitarse a los "climas de referencia" de la Norma Española, se ajusta a la realidad local, ampliando la cobertura de opciones para el diseño bioclimático. Por todo ello, se puede considerar un resultado fiable también desde esta perspectiva.

\section{PUNTO 2 DE DISCUSIÓN: APLICABILIDAD DE LA CARTOGRAFÍA PRESENTADA.}

Una segunda cuestión a discutir sobre el resultado obtenido es si la cartografía obtenida tiene alguna otra aplicabilidad más allá de reflejar las estrategias de diseño necesarias para alcanzar el confort cada mes, según Givoni. Mediante el análisis efectuado en el proceso de investigación, se han evidenciado varias aplicaciones de gran potencialidad.

La primera de ellas es considerar esta cartografía como manual de diseño bioclimático de la región cartografiada. Cualquier técnico o promotor, con la localización de la parcela en el mapa, obtiene de forma directa la información relativa a las estrategias de diseño necesarias de adoptar para alcanzar el confort en cada uno de los meses. Todo esto, de forma directa, sin tener que buscar e interpretar los datos climáticos concretos (en muchos territorios esta búsqueda es de gran complejidad para un técnico particular).

Con la determinación de las estrategias reveladas en los mapas, junto con las condiciones específicas del entorno, el diseñador puede establecer sus propios criterios de diseño, adecuados a la parcela, al proyecto o a los requerimientos del uso o del propio promotor. De tal manera, se fomenta el surgimiento de propuestas de diseño eficientes y optimizadas.

Una segunda aplicación consiste en el conocimiento de la evolución de las necesidades de las propias estrategias en el propio territorio. Mediante la observación de todos los mapas en conjunto, se puede apreciar la evolución de las zonas correspondientes a cada estrategia a lo largo del año.

Del análisis individual por estrategias, en el caso aplicado, se desprende cuáles zonas están en confort, no requiriendo ninguna estrategia incluso, en situación de temperaturas límite. Asimismo, se puede advertir que, en más de la mitad del territorio, no sería necesario disponer de sistemas de aire acondicionado en ningún periodo del año.

A partir del análisis de la evolución de las estrategias en el año, se determina la posibilidad de generar una zonificación climática anual, ajustada a la realidad física de cada territorio (Da Casa et al., 2017), y con ello establecer la coherencia con la normativa aplicable (en su caso, como en España, con el Código Técnico de la Edificación). Se pueden localizar las zonas de singularidad específica, lo que permitiría realizar ajustes concretos en las normativas para evitar la aparición de comportamientos anómalos, al no ajustarse a las características generales normativas.

Una tercera aplicación es su configuración como una herramienta de gran potencial para la investigación del territorio. Por un lado, permite realizar análisis comparativos de las zonas obtenidas, pero también de otros factores, como aquellos dependientes de la intervención humana, entre los cuales puede mencionarse:

- La ubicación de la población humana.

- Las vías de comunicación territorial.

- La arquitectura popular y vernácula del territorio

- El estudio de las necesidades según los usos del territorio.

Por otro lado, permite investigar, mediante análisis comparativo, sobre la relación con los condicionantes impuestos por las características del territorio. Así, por ejemplo, este método abre puertas al estudio de:

- La geología del territorio; con el objetivo de tener conocimiento de los materiales propios de cada área definida y su posible aplicación en el marco de la construcción, como también de las condiciones que la propia naturaleza del terreno impone, afectando de modo directo al microclima local.

- Los aspectos orográficos, topográficos y morfológicos, dadas las condiciones de variabilidad entre costa y alta montaña, y entre distintas altitudes.

- La hidrología, dentro de este ámbito de estudio, ya que influye en el grado de humedad ambiental. La presencia de agua (superficial o subterránea) puede implicar una sensible variación de las estrategias.

- La vegetación del territorio y su variación. Las modificaciones que la vegetación incorpora al microclima local están determinadas por su tamaño y tipología. Entre ellas, las principales serán el aporte de humedad, y una relativa suavidad climática, así como la capacidad de proporcionar sombras y protección del viento. 
- La incidencia del viento en el territorio. Variaciones de pendiente, o relieve, y las obstrucciones existentes pueden alterar los parámetros obtenidos en los observatorios meteorológicos. Los factores que inciden en los cambios de velocidad, dirección y frecuencia de los vientos dominantes afectan a las necesidades de ventilación, protección a viento o a la combinación de ambas, necesarias para conseguir el confort.

- La incidencia de la radiación solar y los valores de incidencia por meses. Se puede determinar la capacidad de captación en los meses menos favorables y la necesidad de su protección en los meses que registran valores máximos.

\section{PUNTO 3 DE DISCUSIÓN: CAPACIDAD DE EXTRAPOLAR LA METODOLOGÍA UTILIZADA A OTROS TERRITORIOS}

El último punto de discusión planteado se pregunta si la metodología propuesta tiene capacidad de ser extrapolada para ser aplicada a otro territorio. Pues bien, tras el desarrollo de la investigación se puede deducir su fácil aplicación a cualquier territorio, independientemente de la extensión del mismo.

Para un correcto uso de esta metodología es, sin embargo, fundamental tener conciencia de sus limitaciones. Hay que tener en cuenta que:

- La primera condición es disponer de suficientes observatorios meteorológicos con los datos que se precisan, con una distribución geográfica homogénea. La disposición geográfica distante entre observatorios puede obviar algunas situaciones intermedias. A mayor número de observatorios, mejora la precisión de la cartografía resultante.

- Al utilizarse datos climáticos que expresan medias mensuales, no se contemplan situaciones extremas (estas pudiesen registrarse, sin embargo, en la etapa de preparación de los datos). No se puede tratar la información obtenida como una instrucción de actuación. La cartografía así obtenida aporta una idea de orden y magnitud de las estrategias en la zona geográfica de estudio, pudiendo diferenciar el comportamiento general de diversas zonas identificadas.

- Los límites de las zonas obtenidas, al constituir agrupaciones de datos, pueden tener un cierto grado de inexactitud. En caso de duda, se debería hacer un análisis en mayor profundidad de las características del entorno.

Estos son aspectos que se plantea mejorar en la progresión del proyecto de investigación del que es fruto este trabajo. En ese sentido, es de reseñar que, para las administraciones públicas de cada territorio, el coste de la obtención de estos datos es mínimo, ya que generalmente se dispone del acceso a dicha información. Esos beneficios se multiplican, en el caso de territorios donde no se cuenta con una normativa específica para la eficiencia energética o en los que no se posee climas de referencia.
La cartografía presentada facilita, por último, su potencial aplicación como manual de diseño bioclimático, para la cual vale una recomendación final. El proceso se iniciaría con la localización de la parcela en el mapa. Se obtiene así de forma directa la información relativa a las estrategias de diseño a adoptar, para alcanzar el confort en cada uno de los meses del año. Se debe considerar los resultados obtenidos como una aproximación a la situación real, por lo que ha de ser el diseñador quien establezca los criterios de diseño y tome las decisiones que considere oportunas, siempre teniendo en cuenta el resto de los parámetros (la parcela, el objeto del proyecto, o los requerimientos del uso o del propio promotor).

\section{CONCLUSIONES}

La principal aportación de la investigación realizada y aquí expuesta es la metodología para elaborar una cartografía regional, cuyo fin consiste en aplicar estrategias bioclimáticas para alcanzar niveles adecuados de confort térmico, siguiendo la carta bioclimática de Givoni.

En el desarrollo del trabajo ha quedado validada la metodología en cuestión a través de su aplicación a un territorio concreto en España (la Comunidad Autónoma de Galicia), al obtenerse como resultado un conjunto de mapas de periodicidad mensual, donde se establecen las estrategias de intervención necesarias, bajo el prisma de los parámetros bioclimáticos, que se deben adoptar para poder alcanzar el confort térmico en los edificios, en dicho territorio y en dicho periodo.

La cartografía así producida se convierte en una potente herramienta para el diseño bioclimático en el territorio estudiado. En consecuencia, las posibilidades de desarrollar arquitectura bioclimática son evidentes. La herramienta, en efecto, constituye un campo abierto y pleno de posibilidades para insertarse en los sistemas habituales de proyectación arquitectónica. El desarrollo y aplicación de estas estrategias contribuye a alcanzar los objetivos ODS, así como de todas las normativas de eficiencia energética, tanto respecto a la reducción de consumos, como al aumento de la optimización térmica de los edificios desde su propio diseño.

La sistemática aquí descrita es susceptible de ser aplicada a cualquier territorio que se considere preciso, más allá de la extensión del mismo. Con ello, se puede, además, disponer de una herramienta para aumentar el conocimiento del propio territorio cartografiado.

\section{AGRADECIMIENTOS}

El desarrollo de esta investigación se realiza dentro del Proyecto ENE2013-48015-C3-2R (Integrated system for energy optimization and reduction of building $\mathrm{CO} 2$ footprint: BIM. Indoor mapping, UAV technologies and advanced energy simulations tools), financiado por el Ministerio de Economía y Competitividad de España. 


\section{REFERENCIAS BIBLIOGRÁFICAS}

Arava, J.L. (2014). Experiencias en la aplicación operativa de un método multivariado de imputación de datos meteorológicos. Tecnología en Marcha, 27(3), 70-79.

Balter, J., Ganem, C., Discoli, C. (2016). Energy savings for the cooling of indoor spaces through the evaluation of solar control systems in high-rise residential buildings: the case of the oasis city of Mendoza, Argentina. Hábitat sustentable, 6(1), 73-83.

Cartaya, S., Zurita, S. y Montalvo, V. (2016). Métodos de ajuste y homogenización de datos climáticos para determinar índice de humedad de Lang en la provincia de Manabí, Ecuador. La Técnica, (16), 94-106.

Corral, J. A. R., García, G. M. y Romero, G. E. G. (2018). Sistema de información agroclimático para México-Centroamérica. Revista Mexicana de Ciencias Agrícolas, 9(1), 1-10.

Couret, D. G., Guzmán, L. A. R., Milián, N. G., García, E. R. y Salazar, M. L. (2015). Evaluación cualitativa de la influencia del diseño arquitectónico en el ambiente interior. Arquitectura y Urbanismo, 36(3), 53-66.

Da Casa, F. (2000). Adecuación bioclimática en la subregión de Madrid para el diseño de los edificios y sus elementos constructivos. Tesis Doctoral. Universidad Politécnica de Madrid (edición digital Biblioteca UPM- ID 563).

Da Casa, F., Echeverría, E. E. y Celis, F. (2017). Zonificación climática para su aplicación al diseño bioclimático. Aplicación en Galicia (España). Informes de la Construcción, 69(547), e218. DOl: http:// dx.doi. org/10.3989/id55319.

Dávila, J. (2015). Arquitectura bioclimática y sustentable basada en los requerimientos del usuario-sitio. Diseño biblioteca pública de Santiago de Querétaro, Querétaro. Semana de la sustentabilidad CyAD. UAM Azcapotzalco.

Díaz, F., Montero, G., Mazorra-Aguiar, L. (2018). Solar Radiation Maps. En Pérez, R. (Ed.), Wind Field and Solar Radiation Characterization and Forecasting (pp. 229-257). Springer, Cham.

Echeverría, E., García, R., Celis, F. y Saelzer, G. (2019). Integrated design experiences for energy-efficient housing in Chile, Construction Innovation, 19(2), 236-255. DOI: https://doi.org/10.1108/Cl-05-20170042

Enríquez, A., Díaz, R., Martín, R. y Santos, M. (2017). Environmental impacts of climate change adaptation. Environmental Impact Assessment Review, 64, 87-96. DOI: 10.1016/j.eiar.2017.03.005.

Esteves, A. (2018). Arquitectura bioclimática y sustentable. Mendoza. Ed: Alfredo Esteves.

Givoni, B. (1969). Climate and architecture. Amsterdam; London; New York: Ed. Elsevier.

Gou, S., Li, Z., Zhao, Q., Nik, V.M. Y Scartezzini, J. (2015). Climate responsive strategies of traditional dwellings located in an ancient village in hot summer and cold winter region of China. Building and environment, 86, 151-165.

Guzmán-Hernández, I. A., Cano, F. y Roset, J. (2019). Problemática de los sistemas pasivos de climatización en zonas tropicales cálidohúmedas. AULA Revista de Humanidades y Ciencias Sociales, 64(4).
Recuperado de https://revistas.unphu.edu.do/index.php/aula/article/ view/104

Halawa, E. y Van Hoof, J. (2012). The adaptive approach to thermal comfort - A critical overview. Energy and Buidlings, 51, 101-110. DOI: 10.1016/j.enbuild.2012.04.011

Houghten, F., Yagloglou, C.P. (1923). Determining lines of equal confort. ASHVE Transactions, 29(10), 163-176.

Kurbán, A. y Cúnsulo, M. (2017). Confort térmico en espacios verdes urbanos de ambientes áridos. Hábitat Sustentable, 7(1), 32-43.

Larrumbide, E. y Bedoya, C. (2015). El comportamiento del hueco de ventana en la arquitectura vernácula mediterránea española ante las necesidades de acondicionamiento solar. Informes de la Construcción, 67(539), 120-130. DOI: http://dx.doi.org/10.3989/ic.14.056.

Luciani-Mejía, S., Velasco-Gómez, R. y Hudson, R. (2018). Eco-friendly coverings: Analysis of the use of ventilated facades in hot, humid weather. Revista de Arquitectura, 20(2), 62-77.

Marco, R., Sariñena, M.A., López, M.S. y López, M.L. (2016). AWisobioclimas: aplicación web para consultar los isobioclimas de la España peninsular y balear. En X Congreso Internacional AEC: Clima, sociedad, riesgos y ordenación del territorio. DOI: http://dx.doi.org/10.14198/ XCongresoAECAlicante2016-20.

Medina Patrón, N. y Escobar Saiz, J. (2019). Envolventes eficientes. Relación entre condiciones ambientales, espacios confortables y simulaciones digitales. Revista de Arquitectura, 21(1), 90-109.

Mena, R., Rodríguez, F., Castilla, M. y Arahal, M.R. (2014). A prediction model based on neural networks for the energy consumption of $\mathrm{CIESOL}$ bioclimatic building. Energy and Buildings, 82, 142-155. DOI: 10.1016/j. enbuild.2014.06.052.

Ooka, R. (2002). Field study on sustainable indoor climate design of a japanese traditional folkhouse in cold climate area. Building and environment, 37, 319-329. DOI: 10.1016/S0360-1323(00)00085-8.

Pérez, J.L., Ladrón de Guevara, I. y Boned, J. (2015). Incidencia del clima local en los procesos de planificación territorial: Análisis bioclimático de la Costa del Sol Occidental de Málaga (España). EURE (Santiago), 41(123) 187-210. DOI: https://dx.doi.org/10.4067/S0250-71612015000300008.

Rodríguez Muñoz, N. A., Nájera Trejo, M. y Martín Domínguez, I. R. (2018). Análisis del desempeño térmico de los sistemas constructivos de un edificio de oficinas mediante simulaciones dinámicas. Ingeniería, investigación y tecnología, 19(3), 279-289.

Rubio-Bellido, C., Pulido-Arcas, J. A., Ureta-Gragera, M. (2015). Aplicabilidad de estrategias genéricas de diseño pasivo en edificaciones bajo la influencia del cambio climático en Concepción y Santiago, Chile. Hábitat Sustentable, 5(2), 33-41.

Sánchez, D., Rubio, C., Marrero, M., Guevara, F.J. y Canivell, J. (2017) El control adaptativo en instalaciones existentes y su potencial en el contexto del cambio climático. Hábitat Sustentable, 7(2), 6-17.

Solanki, S.K., Schüssler, M. y Fligge, M. (2000). Evolution of the Sun's large-scale magnetic field since the Maunder minimum. Nature, 408(6811), 445-7.

Solanki, S.K., Usoskin, I. G., Kromer, B., Schüssler, M. y Beer, J. (2004). Unusual activity of the Sun during recent decades compared to the previous 11.000 years. Nature 431 (7012), 1084-1087 (2004). DOI:10.1038/nature02995 